\title{
On the definition of the covariant lattice Dirac operator
}

\author{
Claude Roiesnel* \\ June 18, 2018 \\ Centre de Physique Théorique, École Polytechnique, CNRS, \\ 91128 Palaiseau cedex, France.
}

\begin{abstract}
In the continuum the definitions of the covariant Dirac operator and of the gauge covariant derivative operator are tightly intertwined. We point out that the naive discretization of the gauge covariant derivative operator is related to the existence of local unitary operators which allow the definition of a natural lattice gauge covariant derivative. The associated lattice Dirac operator has all the properties of the classical continuum Dirac operator, in particular antihermiticy and chiral invariance in the massless limit, but is of course non-local in accordance to the Nielsen-Ninomiya theorem. We show that this lattice Dirac operator coincides in the limit of an infinite lattice volume with the naive gauge covariant generalization of the SLAC derivative, but contains non-trivial boundary terms for finite-size lattices. Its numerical complexity compares pretty well on finite lattices with smeared lattice Dirac operators.
\end{abstract}

CPHT-RR 068-0812

*claude.roiesnel@cpht.polytechnique.fr 


\section{Introduction}

The standard mathematical description of the dynamics of the strong interactions, a description called Quantum ChromoDynamics (QCD), is obtained by writing the partition function of an Euclidean $S U(3)$ quantum gauge field theory interacting with $N_{f} \geq 2$ fermions in the fundamental representation of the gauge group. The partition function of QCD is postulated by analogy with the path integral formalism of Quantum ElectroDynamics which has been shown to be successful with a very high accuracy. Using the rules of Grassmanian integration, the QCD partition function can be formally written as a functional integral over the non-abelian gauge degrees of freedom only,

$$
\mathcal{Z}=\int \mathcal{D} A_{\mu}\left(\prod_{f} \operatorname{det} D_{f}\right) e^{-S_{G}}
$$

The measure of integration in eq. (1) can be interpreted as a formal probability measure over the space of gauge configurations because the Euclidean Dirac operator $D_{f}$ of each fermion flavor is antihermitian (with the right boundary conditions) and chirally invariant in the massless limit,

$$
\begin{array}{r}
D_{f}=\gamma_{\mu} D_{\mu}+m_{f}, \quad D_{\mu}=\partial_{\mu}+i g A_{\mu} \\
\left\{\gamma_{5}, \gamma_{\mu} D_{\mu}\right\}=0, \quad\left\{\gamma_{\mu}, \gamma_{\nu}\right\}=2 \delta_{\mu \nu}, \quad \gamma_{\mu}^{\dagger}=\gamma_{\mu} .
\end{array}
$$

Hence the eigenvalues of each operator $D_{f}$ come in complex conjugate pairs, up to a possible discrete set of zero modes of the operator $\gamma_{\mu} D_{\mu}$, which guarantees reality and positiveness of their determinant for massive fermions, $\operatorname{det} D_{f}>0$.

The Euclidean gauge action (summation over repeated indices is implied throughout),

$$
S_{G}=\frac{1}{4} F_{\mu \nu} F_{\mu \nu}, \quad F_{\mu \nu}=\partial_{\mu} A_{\nu}-\partial_{\nu} A_{\mu}+i g\left[A_{\mu}, A_{\nu}\right]
$$

is invariant under the local gauge transformations $G(x) \in S U(3)$,

$$
A_{\mu}(x) \longrightarrow G(x) A_{\mu}(x) G^{-1}(x)+\frac{i}{g}\left(\partial_{\mu} G(x)\right) G^{-1}(x)
$$

whereas the operator $D_{f}$ transforms covariantly.

Physical observables can then be related to expectation values with respect to the probability measure (1) of certain gauge-invariant matrix elements $\mathcal{O}(A)$ of operators built out of the Dirac operators and their inverses, provided that the formal measure $\mathcal{D} A_{\mu}$ in (1) be given a precise gauge invariant meaning through a constructive procedure.

Section 2 recalls briefly the main properties of Wilson's lattice regularization which is the only constructive proposal known to date [1]. The discretization of space-time in a finite box allows for the non-perturbative calculation of physical observables by means of numerical simulations. The approach has been very successful in describing many features 
of hadronic physics, except for one thing. It proves difficult to reproduce the continuum physics with the physical light quark masses.

The reason is well understood [3] and resides in the formulation of lattice fermions. The Lorentz invariant regularization of quantum fluctuations in continuum QCD generate a chiral anomaly which cannot be duplicated on the lattice with a discretization of the Dirac operator which is local, chirally symmetric and contains the correct number of fermionic degrees of freedom in the continuum limit. This result is usually referred to as the no-go theorem. The standard avoidance is to hold to local fermions and break chiral symmetry explicitly.

In section 3 we reconsider the naive discretization of the covariant derivative and identify a set of local unitary operators which enables the definition of another lattice gauge covariant derivative with the same algebraic properties as in the continuum. This lattice derivative is non-local in accordance to the no-go theorem and coincides in the free limit with the SLAC derivative [4]. The lattice fermion formulation based on the SLAC derivative has been extensively discussed three decades ago. A general consensus has emerged according to which formulations of non-local lattice fermions coupled to gauge fields lead to various inconsistenties in weak coupling perturbation theory $[5,6,7,8,9]$, and cannot reproduce the continuum limit properly, in particular the axial anomaly [10]. In fact we are not aware of a single numerical study of the functional integral of a SLACtype fermion coupled to a compact lattice gauge field.

Nonetheless we think it is fair to state that none of the objections to non-local fermions has the status of a no-go theorem. In all studies to date, the coupling of a SLAC fermion to a gauge field on the lattice has been written by mimicking the textbook derivation of a local gauge symmetry in the continuum. The result is correct only for infinite lattices. The boundary conditions on finite-size lattices are not taken into account by the standard technique. The unitary operators exhibited in section 3 are the right tools to include boundary conditions. As an example, in section 4 we diagonalize these unitary operators on periodic lattices and express in section 5 the matrix elements of the associated lattice Dirac operator in configuration space. We find non-trivial boundary contributions which vanish only in the limit of infinite physical volume.

The constraints of the underlying locality of the exponentiated non-local lattice gauge covariant derivative probably cannot be neglected in analyzing the weak coupling perturbation theory, even in the infinite lattice volume limit. The intent of the present work is not to address this complicated issue, which deserves a separate work, but to stress its existence.

In the concluding remarks we point out that the numerical complexity of the associated finite size lattice Dirac operator is similar to a five-dimensional local Dirac operator. In fact the algorithmic implementation is much simpler. Moreover this non-local Dirac operator can be naturally interpreted as smeared over the Wilson lines. This smearing has the virtue to be completely analytic. Numerical tests of the meaningfulness of the finitesize formulation of non-local fermions coupled to gauge fields can easily be performed on a single desktop computer in the quenched approximation up to four dimensions. 


\section{Lattice regularization}

As is well-known, Wilson's formulation consists of regularizing the Euclidean continuum gauge theory on a finite four-dimensional lattice $\mathcal{L}$ with hypercubic cells of spacing $a$, and of replacing the continuum gauge degrees of freedom, the gauge potential $A_{\mu}(x)$ which belongs to the $S U(3)$ Lie algebra, by variables $U_{x, \mu}$ associated to each link $(x, x+a \widehat{\mu})$ of the lattice and which belong to the $S U(3)$ group manifold. Then eq.(1) becomes

$$
\mathcal{Z}_{\mathcal{L}}=\int\left(\prod_{(x, x+a \widehat{\mu}) \in \mathcal{L}} d U_{x, \mu}\right)\left(\prod_{f} \operatorname{det} M_{f}(U)\right) e^{-S_{G}(U)} .
$$

where the integration measure is now a perfectly well-defined finite product of gaugeinvariant Haar measures over the $S U(3)$ group manifold, and $S_{G}(U)$ and $M_{f}(U)$ are discretized versions of the continuum gauge action and Dirac operators. This measure can be evaluated numerically by stochastic importance sampling.

There is a large arbitrariness in the choice of lattice gauge action and lattice Dirac operators. The main constraint is that the lattice regularized model possess a second-order critical point which reproduces the asymptotic freedom of QCD in the continuum limit, with critical exponents predicted by perturbation theory. This requires in particular that the lattice operators reproduce the naive continuum definitions when the lattice spacing vanishes, $a \rightarrow 0$. Scaling theory then suggests that there exists a whole universality class of lattice actions which correspond to different regularizations of the same continuum theory and whose critical properties are related by renormalization group transformations.

The most direct ab-initio approach is to consider lattice QCD actions with the same number of parameters as continuum QCD. The simplest such lattice gauge action, the Wilson action [1], has discretization errors of $\mathcal{O}\left(a^{2}\right)$,

$$
\begin{aligned}
& S_{w}(U)=\beta \sum_{x, \mu<\nu}\left(1-\frac{1}{6} \operatorname{Tr}\left(P_{\mu \nu}(x)+P_{\mu \nu}^{\dagger}(x)\right)\right), \\
& \text { with } \quad P_{\mu \nu}(x)=U_{x, \mu} U_{x+a \widehat{\mu}, \nu} U_{x+a \widehat{\nu}, \mu}^{\dagger} U_{x, \nu}^{\dagger}, \quad \beta=\frac{6}{g^{2}} .
\end{aligned}
$$

Local gauge invariance is preserved on the lattice provided that the variables $U_{x, \mu}$ transform as

$$
U_{x, \mu} \longrightarrow G(x) U_{x, \mu} G^{-1}(x+a \widehat{\mu}) .
$$

The simplest candidate for a lattice Dirac operator is expressed in terms of the naive discretization of the covariant derivative operator, namely,

$$
\begin{aligned}
\mathcal{D}_{l}(U) & =\gamma_{\mu} D_{l, \mu}(U)+m \\
\left(D_{l, \mu}(U) \psi\right)_{x} & =\frac{1}{a}\left(U_{x, \mu} \psi_{x+a \widehat{\mu}}-\psi_{x}\right),
\end{aligned}
$$


which has the correct covariant transformation law under (7) but does not have a spectrum with definite transformation properties under the conjugation operation. Hence the determinant $\operatorname{det} \mathcal{D}_{l}(U)$ is complex in general and does not define a probability measure.

An obvious workaround would be to introduce antihermitian covariant difference operators,

$$
\left(D_{s, \mu}(U) \psi\right)_{x}=\frac{1}{2 a}\left(U_{x, \mu} \psi_{x+a \widehat{\mu}}-U_{x-a \widehat{\mu}, \mu}^{\dagger} \psi_{x-a \widehat{\mu}}\right)
$$

which have the same conjugation properties as the continuum operators and produces a valid probability measure. But the operator $\gamma_{\mu} D_{s, \mu}$ is plagued by the famous fermion doubling problem, due to the use of a central difference operator, and does not describe a single fermion flavor even in the continuum limit.

Wilson proposed [2] to add to $D_{s}$ a piece proportional to the finite difference approximation to the Laplacian operator $\Delta$, which lifts the mass degeneracy of the fermion doublers by terms of order $1 / a$ at the expense of breaking chiral invariance explicitly. However the Wilson operator still possess the same pseudo-hermiticity property as the continuum Dirac operator,

$$
\mathcal{D}_{w}(U)=\gamma_{\mu} D_{s, \mu}(U)-r \Delta_{L} \quad(0<r \leq 1), \quad \mathcal{D}_{w}^{\dagger}=\gamma_{5} \mathcal{D}_{w} \gamma_{5},
$$

which guarantees the invariance of its spectrum under conjugacy and the interpretation of (5) as a probability measure. It was later realized [3] that it is not possible to devise a (ultra-)local lattice Dirac operator with the correct classical continuum limit and without fermion doublers while preserving exact chiral invariance on the lattice in the massless case.

Various alternative lattice Dirac operators have been put forward during the subsequent three decades, some of which with a presently viable ecosystem. The reader can find all references in a recent, and very nice, review [11] of the state-of-the-art of numerical simulations of lattice gauge theories.

\section{A chirally invariant lattice Dirac operator}

If one examines the definition (8) of the naive lattice covariant derivative operator, one realizes immediately that the four operators,

$$
S_{\mu}=\mathbf{1}+a D_{l, \mu}, \quad\left(S_{\mu}\right)_{i x, j y}=\left(U_{x, x+a \widehat{\mu}}\right)_{i j} \delta_{y, x+a \widehat{\mu}},
$$

are unitary ladder operators which translate by one lattice unit in direction $\widehat{\mu}$ each slice of the lattice field they act upon, while rotating locally their color degrees of freedom,

$$
S_{\mu} S_{\mu}^{\dagger}=S_{\mu}^{\dagger} S_{\mu}=\mathbf{1}, \quad \forall \mu .
$$

The set of operators $S_{\mu}$ transforms covariantly under the local gauge transformations (7),

$$
S_{\mu} \longrightarrow \mathcal{G} S_{\mu} \mathcal{G}^{-1}, \quad(\mathcal{G})_{i x, j y}=G(x)_{i j} \delta_{x y}, \quad \forall \mu
$$


and encodes all the space-time and color degrees of freedom of a gauge field configuration on a four-dimensional lattice. For instance, the Wilson action (6) can be written, up to a constant term, as

$$
S_{w}(U)=-\frac{\beta}{6} \operatorname{Tr}\left(S_{\mu} S_{\nu} S_{\mu}^{\dagger} S_{\nu}^{\dagger}\right)
$$

Expressing the unitary operators $S_{\mu}(U)$ as exponentials of antihermitian operators $D_{r, \mu}(U)$,

$$
S_{\mu}(U)=e^{a D_{r, \mu}(U)}, \quad D_{r, \mu}+D_{r, \mu}^{\dagger}=0, \quad \forall \mu,
$$

singles out the operators $D_{r, \mu}(U)$ as the natural definition of the lattice covariant derivative. Indeed the symmetric covariant difference operators (9) are just the leading approximation in the series expansion of these exponentials with respect to the lattice spacing $a$,

$$
a D_{s, \mu}(U)=\frac{1}{2}\left(e^{a D_{r, \mu}}-e^{-a D_{r, \mu}}\right)
$$

Then we can define the lattice Dirac operator

$$
\mathcal{D}_{r}(U)=\gamma_{\mu} D_{r, \mu}(U)+m
$$

which is antihermitian, chirally symmetric in the massless limit, and transforms covariantly under the local gauge transformations (7). The eigenvalues of the operator $\mathcal{D}_{r}$ come in complex conjugate pairs, $m \pm i \lambda$, up to a possible set of zero modes for the imaginary part which ensures, like in the continuum, reality and positiveness of the determinant for massive fermions, $\operatorname{det} \mathcal{D}_{r}>0$.

The operator $\mathcal{D}_{r}(U)$ is non-local since each lattice covariant derivative operator $D_{r, \mu}$ is a series expansion in the local covariant derivative operator $D_{l, \mu}$,

$$
\begin{aligned}
a D_{r, \mu} & =\log \left(\mathbf{1}+a D_{l, \mu}\right) \\
& =\sum_{n=1}^{+\infty} \frac{(-1)^{n+1} a^{n}}{n} D_{l, \mu}^{n} .
\end{aligned}
$$

From the convergence properties of the series expansion (18), the prospect of a practical numerical implementation of the lattice Dirac operator $\mathcal{D}_{r}$ might seem very slim.

On the other hand, in the free case, the eigenvectors of the operators $S_{\mu}$ are just plane waves and their $3 N^{3}$ degenerate spectrum is simply

$$
\operatorname{Spec} S_{\mu}(\mathbf{1})=\left\{e^{i a p_{\mu}}, p_{\mu}=\frac{2 \pi n}{a N_{\mu}},-\frac{N_{\mu}}{2} \leq n<\frac{N_{\mu}}{2}\right\} .
$$

In this limit the lattice Dirac operator $\mathcal{D}_{r}$ has a discrete Fourier representation which has the same form as in the continuum,

$$
\widehat{\mathcal{D}}_{r}(\mathbf{1})=i \gamma_{\mu} p_{\mu}+m
$$


which shows that the operator $\mathcal{D}_{r}$ is not afflicted with the fermion doubling problem. In compliance to the Nielsen-Ninomya theorem, the price to pay is the non-locality of the operator $\mathcal{D}_{r}(U)$. The operator $D_{r, \mu}(\mathbf{1})$ coincides with the SLAC derivative introduced long ago [4] which reads, in the limit of an infinite lattice volume,

$$
D_{\infty, \mu}(x-y)=\int_{-\pi / a}^{\pi / a} \frac{d^{4} p}{(2 \pi)^{4}} i p_{\mu} e^{i p \cdot(x-y)} .
$$

The usual recipe to couple a SLAC-type fermion to a $S U(3)$ gauge field consists in restoring gauge invariance by inserting, if $x-y$ has a non-vanishing component only in direction $\widehat{\mu}$, the Wilson $S U(3)$ ordered straight line integral $W_{\mu}(U, x, y)$ between $x$ and $y$,

$$
\begin{gathered}
\left(D_{S}(U)\right)_{\alpha i x, \beta j y}=m \delta_{\alpha \beta} \delta_{i j} \delta_{x y}+\sum_{\mu}\left(\gamma_{\mu}\right)_{\alpha \beta} D_{\infty, \mu}(x-y)\left(W_{\mu}(U, x, y)\right)_{i j}, \\
W_{\mu}(U, x, y)=\delta_{\mathbf{x}_{\mu}^{\perp}, \mathbf{y}_{\mu}^{\perp}} \prod_{k=0}^{\left(y_{\mu}-x_{\mu}-1\right) / a} U_{x+k a \widehat{\mu}, x+(k+1) a \widehat{\mu}}, \quad \text { if } y_{\mu}>x_{\mu} .
\end{gathered}
$$

where $x=\left(\mathbf{x}_{\mu}^{\perp}, x_{\mu}\right), y=\left(\mathbf{y}_{\mu}^{\perp}, y_{\mu}\right)$, and $\mathbf{x}_{\mu}^{\perp}, \mathbf{y}_{\mu}^{\perp}$ label the sites in the three-dimensional slices orthogonal to the $\mu$ th direction. If $y_{\mu}<x_{\mu}$, we have of course $W_{\mu}(U, x, y)=W_{\mu}(U, y, x)^{\dagger}$.

The matrix elements (22) are certainly correct in the limit of an infinite lattice. However the boundary conditions on finite-size lattices are not taken into account by the conventional prescription. The operators $S_{\mu}$ have such a simple structure that it makes possible their explicit diagonalization for an arbitrary background lattice gauge field configuration and arbitrary boundary conditions. We shall find non-trivial boundary terms in the matrix elements of the operator $\mathcal{D}_{r}$ on finite-size lattices.

To the best of our knowledge, the underlying local unitary structure of the lattice gauge covariant generalization of the SLAC derivative does not seem to have been appreciated since its introduction.

\section{Explicit diagonalization of the unitary operators}

For definiteness we shall assume the lattice to be hypercubic, $N_{1}=N_{2}=N_{3}=N_{4} \equiv N$, and we shall impose periodic boundary conditions on both the lattice gauge field configuration and the lattice matter fields which can be scalars, fermions, $\cdots$. The operators $S_{\mu}$ do not commute in general and have different eigenspectra $\left\{\lambda_{\mu}, \psi_{\lambda_{\mu}}\right\}$. Iterating the eigenvalue equation for $S_{\mu}$,

$$
S_{\mu} \psi_{\lambda_{\mu}}=\lambda_{\mu} \psi_{\lambda_{\mu}}
$$

yields

$$
\begin{aligned}
\left(S_{\mu}^{n} \psi_{\lambda_{\mu}}\right)_{i x} & =\left(\prod_{k=0}^{n-1} U_{x+k a \widehat{\mu}, x+(k+1) a \widehat{\mu}}\right)_{i j}\left(\psi_{\lambda_{\mu}}\right)_{j, x+n a \widehat{\mu}} \\
& =\lambda_{\mu}^{n}\left(\psi_{\lambda_{\mu}}\right)_{i x} .
\end{aligned}
$$


Imposing the periodic boundary condition,

$$
\psi_{x+N a \widehat{\mu}}=\psi_{x}, \quad \forall x,
$$

implies that the eigenvectors $\psi_{\lambda_{\mu}}$ satisfy the equations

$$
\left(W_{\mu}(U, x, x+N a \widehat{\mu})\right)_{i j}\left(\psi_{\lambda_{\mu}}\right)_{j x}=\lambda_{\mu}^{N}\left(\psi_{\lambda_{\mu}}\right)_{i x}, \quad \forall x
$$

where $W_{\mu}(U, x, x+N a \widehat{\mu})$, defined in $(23)$, is the Wilson line from $x$ in direction $\widehat{\mu}$ which wraps the lattice. We shall use the shorthand $W_{\mu, x}(U)$ for such Wilson lines and the argument $U$ will be implicit most of the time.

Hence each non-zero space-time component of the eigenvector $\psi_{\lambda_{\mu}}$ is a color triplet which is an eigenvector of some Wilson line $W_{\mu, x}(U)$. The Wilson lines are covariant objects under the local gauge transformations (7) and their eigenvalues are gauge-invariant and do not depend on the choice of base points $x$ which differ only by the $x_{\mu}$ component along their direction. Indeed a change of base point along a Wilson line is nothing but a similarity transformation.

Therefore the eigenspectra of the Wilson lines $W_{\mu, x}(U)$ can be labelled by the points $\vec{x}$ of the lattice slice $x_{\mu}=0, \vec{x} \equiv\left(\mathbf{x}_{\mu}^{\perp}, 0\right)$,

$$
\left\{e^{i \delta_{\mu, \vec{x}}^{c}}, \eta_{\mu, \vec{x}}^{c}\right\}, \quad-\pi<\delta_{\mu, \vec{x}}^{c} \leq \pi, \quad c=1,2,3 .
$$

where $\eta_{\mu, \vec{x}}^{c}$ are the color triplet eigenvectors of $W_{\mu, \vec{x}}(U)$. Their calculation requires only $4 N^{4} S U(3)$ matrix multiplications and $4 N^{3} S U(3)$ matrix diagonalizations.

Barring accidental degeneracies, the eigenvalues $\lambda_{\mu}$ and eigenvectors $\psi_{\lambda_{\mu}}$ fall into families labelled by the $4 \times 3 \times N^{3}$ eigenvalues of the Wilson lines and defined by the equations,

$$
\lambda_{\mu}^{N}=e^{i \delta_{\mu, \vec{x}}^{c}}
$$

The general solution for $\lambda_{\mu}$ reads

$$
\lambda_{\mu, \vec{x}, p_{\mu}}^{c}=e^{i\left(a p_{\mu}+\delta_{\mu, \vec{x}}^{c} / N\right)}, \quad p_{\mu}=\frac{2 \pi n}{N a}-\frac{\pi}{a}, \quad 0 \leq n<N
$$

The $N$ non-vanishing components of the corresponding eigenvector $\psi_{\mu, \vec{x}, p_{\mu}}^{c}$ are, with $n=$ $0, \cdots, N-1$,

$$
\begin{aligned}
\left(\psi_{\mu, \vec{x}, p_{\mu}}^{c}\right)_{j y} & =\left(\lambda_{\mu, \vec{x}, p_{\mu}}^{c}\right)^{n}\left(\eta_{\mu, y}^{c}\right)_{j} \delta_{y, \vec{x}+n a \widehat{\mu}}, \\
\text { with } \quad \eta_{\mu, \vec{x}+y_{\mu} \widehat{\mu}}^{c} & =W_{\mu}^{\dagger}\left(\vec{x}, \vec{x}+y_{\mu} \widehat{\mu}\right) \eta_{\mu, \vec{x}}^{c} .
\end{aligned}
$$

These components can be computed sequentially and the calculation of each of the $12 N^{4}$ eigenvectors requires only $N S U(3)$ matrix-vector multiplications. So the total computational complexity of the complete diagonalization of every operator $S_{\mu}$ is of order $\mathcal{O}\left(N^{5}\right)$. A complete diagonalization has to be performed only once for each lattice gauge field 
configuration and its storage requirement is in practice proportional to the lattice volume since it is more efficient to recompute the $N$ components of each eigenvector when needed.

The eigenvectors of $S_{\mu}$ are also eigenvectors of $D_{r, \mu}$ and the action of $D_{r, \mu}$ on an eigenvector $\psi_{\mu, \vec{x}, p_{\mu}}^{c}$ has a remarkably simple continuum-like expression,

$$
a D_{r, \mu} \psi_{\mu, \vec{x}, p_{\mu}}^{c}=i\left(a p_{\mu}+\bar{\alpha}_{\mu, \vec{x}}^{c}\right) \psi_{\mu, \vec{x}, p_{\mu}}^{c}, \quad \bar{\alpha}_{\mu, \vec{x}}^{c}=\frac{\delta_{\mu, \vec{x}}^{c}}{N}
$$

There is an inherent ambiguity in the logarithmic definition of $D_{r, \mu}$. We have defined rather arbitrarily the phases $\delta_{\mu, \vec{x}}^{c}$ as the principal argument of the eigenvalues of Wilson lines. Other prescriptions are possible for unitary gauge groups.

\section{$5 \quad$ Matrix elements of the operator $\mathcal{D}_{r}(U)$}

The kernel operation which enters most algorithms involving fermions, such as the calculation of the fermion propagator, is the action of the lattice Dirac operator on an arbitrary lattice fermion field. The action of $\mathcal{D}_{r}$ on a fermion $\Psi$ can be written spin-component-wise as

$$
\left(\mathcal{D}_{r}(U) \Psi\right)_{\alpha}=\left(\gamma_{\mu}\right)_{\alpha \beta} D_{r, \mu}(U) \Psi_{\beta}+m \Psi_{\alpha} .
$$

To perform this calculation we just need to expand each spin component of the fermion field over the complete eigensystem of every operator $D_{r, \mu}$,

$$
\Psi_{\beta}=\sum_{c, \vec{x}, p_{\mu}} C_{\beta, \mu, \vec{x}, p_{\mu}}^{c} \psi_{\mu, \vec{x}, p_{\mu}}^{c}, \quad \forall \mu .
$$

We get $4 \times 4 \times 3 \times N^{4}$ equations, with $x=\left(\vec{x}, x_{\mu}\right)$ and $a=1$ throughout this section,

$$
\left(\Psi_{\beta}\right)_{j x}=\sum_{c, p_{\mu}} C_{\beta, \mu, \vec{x}, p_{\mu}}^{c}\left(\lambda_{\mu, \vec{x}, p_{\mu}}^{c}\right)^{x_{\mu}}\left(\eta_{\mu, x}^{c}\right)_{j} .
$$

We can always choose all color triplet eigensystems $\left\{\eta_{\mu, \vec{x}}^{c}\right\}$ to be orthonormal. Then we observe that all eigensystems $\left\{\eta_{\mu, x}^{c}\right\}$ along the same Wilson line are simultaneously orthonormal,

$$
\sum_{j}\left(\eta_{\mu, x}^{\star a}\right)_{j}\left(\eta_{\mu, x}^{b}\right)_{j}=\delta^{a b}, \quad \forall x=\left(\vec{x}, x_{\mu}\right)
$$

since they are related by unitary transformations which preserve the scalar product. Thus we can transform each equation (35) into a simple one-dimensional Fourier series,

$$
\begin{aligned}
\left(\Psi_{\beta}^{\prime c}\right)_{x} & =e^{-i x_{\mu} \bar{\alpha}_{\mu, \vec{x}}^{c}} \sum_{j}\left(\eta_{\mu, x}^{\star c}\right)_{j}\left(\Psi_{\beta}\right)_{j x}, \\
& =\sum_{p_{\mu}} C_{\beta, \mu, \vec{x}, p_{\mu}}^{c} e^{i p_{\mu} x_{\mu}}, \quad \forall x=\left(\vec{x}, x_{\mu}\right) .
\end{aligned}
$$


Therefore the coefficients $C_{\beta, c, \mu, \vec{x}, p_{\mu}}$ are the one-dimensional inverse discrete Fourier transforms,

$$
C_{\beta, \mu, \vec{x}, p_{\mu}}^{c}=\frac{1}{N} \sum_{x_{\mu}}\left(\Psi_{\beta}^{\prime c}\right)_{x} e^{-i p_{\mu} x_{\mu}}
$$

It follows that the total computational complexity of the action of the operator $\mathcal{D}_{r}(U)$ on a fermion field is of order $\mathcal{O}\left(N^{5}\right)$ which is only a factor $N$ more expensive than the action of a a local operator like the Wilson operator $\mathcal{D}_{w}(U)$.

Plugging (38) into (34) yields, with $x=\vec{x}+x_{\mu} \widehat{\mu}$,

$$
\left(D_{r, \mu} \Psi_{\beta}\right)_{j y}=\frac{1}{N} \sum_{c, \vec{x}, x_{\mu}, p_{\mu}} i\left(p_{\mu}+\bar{\alpha}_{\mu, \vec{x}}^{c}\right) e^{-i\left(p_{\mu}+\bar{\alpha}_{\mu, \vec{x}}^{c}\right) x_{\mu}}\left(\sum_{k}\left(\eta_{\mu, x}^{\star c}\right)_{k}\left(\Psi_{\beta}\right)_{k x}\right)\left(\psi_{\mu, \vec{x}, p_{\mu}}^{c}\right)_{j y}
$$

Inserting (31) gives, with $y=\vec{y}+y_{\mu} \widehat{\mu}$,

$$
\left(D_{r, \mu} \Psi_{\beta}\right)_{j y}=\frac{i}{N} \sum_{c, \vec{x}, x_{\mu}, p_{\mu}} \delta_{\vec{y}, \vec{x}}\left(p_{\mu}+\bar{\alpha}_{\mu, \vec{x}}^{c}\right) e^{i\left(p_{\mu}+\bar{\alpha}_{\mu, \vec{x}}^{c}\right)\left(y_{\mu}-x_{\mu}\right)}\left(\sum_{k}\left(\eta_{\mu, x}^{\star c}\right)_{k}\left(\Psi_{\beta}\right)_{k x}\right)\left(\eta_{\mu, y}^{c}\right)_{j}
$$

The summation over $p_{\mu}$ brings in the finite-size SLAC derivative,

$$
D_{N, \mu}\left(x_{\mu}\right)=\frac{1}{N} \sum_{p_{\mu}} i p_{\mu} e^{i p_{\mu} x_{\mu}}
$$

and the summation over $\vec{x}$ produces,

$$
\begin{aligned}
\left(D_{r, \mu} \Psi_{\beta}\right)_{j y} & =\delta_{\vec{x}, \vec{y}} \sum_{x_{\mu}} D_{N, \mu}\left(y_{\mu}-x_{\mu}\right) \sum_{k}\left(\sum_{c} e^{i \bar{\alpha}_{\mu, \vec{y}}^{c}\left(y_{\mu}-x_{\mu}\right)}\left(\eta_{\mu, x}^{\star c}\right)_{k}\left(\eta_{\mu, y}^{c}\right)_{j}\right)\left(\Psi_{\beta}\right)_{k x} \\
& +i \sum_{k}\left(\sum_{c} \bar{\alpha}_{\mu, \vec{y}}^{c}\left(\eta_{\mu, y}^{\star c}\right)_{k}\left(\eta_{\mu, y}^{c}\right)_{j}\right)\left(\Psi_{\beta}\right)_{k y}
\end{aligned}
$$

The last step is to insert (31) and use the identity,

$$
\left(W_{\mu}^{\dagger}\left(\vec{y}, \vec{y}+y_{\mu} \widehat{\mu}\right)\right)_{j l}\left(\sum_{c} f\left(e^{i \delta_{\mu, \vec{y}}^{c}}\right)\left(\eta_{\mu, \vec{y}}^{c}\right)_{l}\left(\eta_{\mu, \vec{y}}^{\star c}\right)_{m}\right)\left(W_{\mu}\left(\vec{y}, \vec{y}+y_{\mu} \widehat{\mu}\right)\right)_{m k}=\left(f\left(W_{\mu, y}\right)\right)_{j k} .
$$

Collecting everything together, the matrix elements of the operator $\mathcal{D}_{r}(U)$ read finally,

$$
\begin{aligned}
\left(\mathcal{D}_{r}(U)\right)_{\alpha j y, \beta i x} & =\delta_{x y}\left(m \delta_{\alpha \beta} \delta_{i j}+\frac{1}{N a} \sum_{\mu}\left(\gamma_{\mu}\right)_{\alpha \beta}\left(\log \left(W_{\mu, y}(U)\right)\right)_{j i}\right)+\delta_{\vec{x}, \vec{y}} \times \\
& \times \sum_{\mu}\left(\gamma_{\mu}\right)_{\alpha \beta} D_{N, \mu}\left(y_{\mu}-x_{\mu}\right)\left(W_{\mu}(U, y, x)\left(W_{\mu, x}(U)\right)^{\left(y_{\mu}-x_{\mu}\right) / N a}\right)_{j i},
\end{aligned}
$$


where we reintroduced the lattice spacing $a$ to make apparent the physical dimensions. The covariance of the operator $\mathcal{D}_{r}(U)$ under the local gauge transformations $(7)$ is clearly satisfied,

$$
\mathcal{D}_{r}(U) \quad \longrightarrow \quad \mathcal{G} \mathcal{D}_{r}(U) \mathcal{G}^{-1}
$$

We find two additional boundary contributions with respect to the infinite volume expression (22). The first one is a diagonal term in configuration space which vanishes proportionally to the inverse physical lattice size. The second one is an insertion in the open Wilson line $W_{\mu}(U, y, x)$ of the closed Wilson line $W_{\mu, x}(U)$ raised to a power the variation of which is also proportional to the distance $y-x$ in physical units. The insertion point can be covariantly transported anywhere along the closed Wilson line.

\section{Outlook}

As recalled in the introductory section, the lattice fermion formulation based on the SLAC derivative has been rather controversial. There have been one-loop calculations with the SLAC operator (22) in weak coupling perturbation theory of lattice Quantum ElectroDynamics (QED) in four dimensions, which have shown the occurence of singularities in the fermion triangle graph [5] and in the vacuum polarization [6], that lead to non-local, non Lorentz covariant expressions. These singularities are generated by the discontinuities of the SLAC derivative at the edges of the Brillouin zone, $p_{\mu}= \pm \pi / a$. It has been claimed [7] that these divergences could not be renormalized while keeping in the continuum limit $a \rightarrow 0$ (at $L=N a \rightarrow \infty$ ), both chiral invariance without extra states and Lorentz invariance. However it has also been suggested [8] that QED could be recovered in the continuum limit by a proper, non-perturbative, treatment of the infrared singularities and by imposing a finite number of non-local renormalization conditions. But the application of such empirical prescriptions to the lattice Schwinger model, namely two-dimensional Quantum Electrodynamics with massless fermions, which is a completely solvable model in the continuum [12], has still generated a spectrum doubling, a vanishing anomaly, a vanishing vacuum expectation value for $\langle\bar{\psi} \psi\rangle$, and a non-covariant axial-vector [9].

Despite all these negative results, a rigorous treatment of the perturbative expansion of the lattice Dirac operator (22) in the infinite volume limit is still lacking. As already emphasized, the underlying unitarity structure of the non-local lattice covariant derivative has not been taken into account in existing studies and the issue of spectrum doubling in such a formulation, which depends crucially upon the handling of singularities in the infinite volume limit, has to be settled accordingly.

Whatever the outcome, we advocate a pragmatic approach, à-la Wilson. We have exhibited a covariant and chirally-invariant lattice Dirac operator on finite-size lattices which has certainly no spectrum doubling in the free limit. Moreover, the expressions (44) of the matrix elements of the operator $\mathcal{D}_{r}$ are quite convenient for an actual computer implementation. If spectrum doubling does occur when the gauge interaction is turned on, one could always add an explicit chiral symmetry breaking term. Our derivation reveals 
that the non-local operator $\mathcal{D}_{r}$ is a smeared operator with a controllable analytic averaging over the links of Wilson lines (whereas the original smearing proposal [13], as well as its many variants, are empirical thickenings of the links). Smeared operators are smoother and it is widely known [11] that their inversion has better convergence properties than local operators. So the accelerated convergence near the chiral limit, whose qualitative nature can be studied in the quenched approximation, may even turn out to compensate for the additional computational complexity of $\mathcal{D}_{r}$ with respect to local Dirac operators such as $\mathcal{D}_{w}$.

\section{References}

[1] K. G. Wilson, Phys. Rev. D10 (1974) 2445.

[2] K. G. Wilson, "Quarks and Strings on a Lattice", Proceedings of the International School of Subnuclear Physics, Erice (1975), CLNS-321.

[3] H. B. Nielsen and M. Ninomiya, Nucl. Phys. B185 (1981) 20.

[4] S. D. Drell, M. Weinstein, S. Yankielowicz, Phys. Rev. D24 (1976) 1627.

[5] L. Karsten and J. Smit, Nucl. Phys. B144 (1978) 536.

[6] L. Karsten and J. Smit, Phys. Lett. 85B (1979) 100.

[7] L. Karsten and J.Smit, Nucl. Phys. B183 (1981) 103.

[8] J. M. Rabin, Phys. Rev. D24 (1981) 3218.

[9] G. T. Bodwin and E. V. Kovacs, Phys. Rev. D35 (1987) 3198.

[10] M. Ninomya and C.-I. Tan, Phys. Rev. Lett. 53 (1984) 1611.

[11] Z. Fodor and C. Hoelbling, Rev. Mod. Phys. 84 (2012) 449.

[12] J. Schwinger, Phys. Rev. 128 (1962) 2425.

[13] M. Albanese et al. (APE Collaboration), Phys. Lett. B192 (1987) 163. 